IV

Vulnerabilität und Spiritual Care 


\title{
Vulnerabilität in der Krise
}

\author{
Martina Schmidhuber
}

\section{Die Vulnerabilität des Mensch-Seins}

Vulnerabilität ist ein Teil der conditio humana. Sehr schnell können sich an und für sich gesunde Menschen in einer vulnerablen Situation wiederfinden. Grundsätzlich sind drei Formen der Vulnerabilität zu unterscheiden, wie dies die drei US-amerikanischen feministischen Philosophinnen Catriona Mackenzie, Wendy Rogers und Susan Dodds (2014) in ihrer Taxonomie plausibel ausführen: inhärent, situativ und pathogen. (1) Menschen sind aufgrund ihrer conditio humana inhärent vulnerabel. Hunger, Durst, Schlafentzug, soziale Isolation - all diese basalen menschlichen Bedürfnisse verweisen auf die inhärente Vulnerabilität des Mensch-Seins. (2) Die situative Vulnerabilität kann Menschen in bestimmten Kontexten treffen, aus persönlichen, sozialen oder politischen Gründen. Sowohl eine Naturkatastrophe, die von heute auf morgen Menschen obdachlos werden lassen kann, als auch Krankheiten, die das scheinbar starke Subjekt zum hilfsbedürftigen Pflegefall machen, oder plötzliche Arbeitslosigkeit sind Formen situativer Vulnerabilität. (3) Pathogene Vulnerabilität entsteht durch asymmetrische interpersonelle Beziehungen und institutionelle Strukturen. Dazu zählen beispielsweise die Misshandlung oder der Missbrauch eines Kindes durch einen Erwachsenen.

Eine Pandemie wie die Corona-Krise führt Menschen insbesondere ihre situative Vulnerabilität vor Augen, aber auch die inhärente durch soziale Isolation. Und auch der pathogenen Vulnerabilität sind v.a. Kinder und Frauen in Familien, in denen - bisher vielleicht nur latente - Gewalt ein Thema war, in der Krise aufgrund der Ausgangsbeschränkungen besonders ausgesetzt. Menschen spüren Formen der Vulnerabilität aus verschiedenen Gründen in unterschiedlichen Lebenslagen. Nur einige Situationen und Gegebenheiten, die Vulnerabilität in der Krise spürbar machen, ohne Anspruch auf Vollständigkeit, sind folgende: die Erkrankung an Covid-19; eine chronische Erkrankung und/oder ein höheres Alter und dadurch die besondere Gefährdung, ein/e intensivmedizinische/r PatientIn zu werden; der Verlust des Arbeitsplatzes aufgrund der mit Covid-19 einhergehenden wirtschaftlichen Krise, damit verbundene Probleme, die Miete zu bezah- 
len, und in weiterer Folge Existenzängste; das Verbot des Treffens nahestehender Menschen, die in einem anderen Land leben, und damit verbundene Einsamkeit; das Verbot, den wöchentlichen Sportkurs zu besuchen, und eine damit einhergehende soziale Isolation als Alleinlebende/r; die Unmöglichkeit, in die Kirche gehen zu können, und der dadurch erlittene Verlust eines wichtigen spirituellen Rituals im Leben. Selbstverständlichkeiten des Alltags vor der Krise brechen plötzlich weg und legen die menschliche Vulnerabilität in diversen Facetten offen. Menschen, die sich stets in vulnerablen Situationen befinden, etwa aufgrund einer chronischen Erkrankung und/oder wegen ihres höheren Alters, sind besonders betroffen und ihre ohnehin schon bestehende Vulnerabilität wird noch verstärkt.

Im vorliegenden Beitrag soll es deshalb einerseits darum gehen, den Blick auf eine besonders vulnerable Gruppe in der Pandemie zu lenken, nämlich Menschen mit Demenz und ihre Angehörigen, und andererseits soll auch gezeigt werden, dass das schrittweise wirtschaftliche und gesellschaftliche Wiederhochfahren eines Landes kein Widerspruch zum Schutz der vulnerablen Gruppen ist. Im Gegenteil: Um nicht noch mehr Vulnerabilität aufgrund von Arbeitslosigkeit und Existenzgefährdung hervorzurufen, ist es sogar geboten, die vulnerablen Gruppen gesondert in den Blick zu nehmen und ihnen besondere Betreuung und Fürsorge zu gewährleisten. Wie an den Beispielen oben gezeigt wurde, entsteht Vulnerabilität nicht allein aufgrund von Krankheit, sondern kann sich auch in existenzieller, ökonomischer, sozialer und spiritueller Hinsicht äußern. Es gilt, Menschen, die nicht gesundheitlich gefährdet sind, wieder ein gutes Leben zu ermöglichen (Nussbaum 1999), indem sie wieder in die Arbeit gehen dürfen, Freunde und Familie treffen, ihren Sportverein oder den Gottesdienst besuchen können.

\section{Menschen mit Demenz und ihre Angehörigen in der Krise}

Wenn im Folgenden Menschen mit Demenz in ihrer Vulnerabilität im Fokus stehen, ist es erforderlich, auch stets ihre Angehörigen in den Blick zu nehmen. Denn sie sind es, die diese demenziellen Persönlichkeitsveränderungen der primär Betroffenen am meisten miterleben. Pflegende Angehörige sind deshalb sekundär betroffen (Gräßel/Niefanger 2012).

Menschen mit Demenz sind in unserer Gesellschaft schon grundsätzlich eine vulnerable Gruppe. Sie fallen auf durch ihre Langsamkeit, ihre Vergesslichkeit und ihre Orientierungslosigkeit in Bezug auf Ort und Zeit. In der Schnelllebigkeit, der sich unsere westliche Gesellschaft zum Großteil 
verschrieben hat, haben sie nur am Rande Platz. Das Plädoyer Reimer Gronemeyers (2013), sie in die Mitte der Gesellschaft zu holen, scheint kaum durchführbar, wenngleich es auch von einigen Seiten Bemühungen gibt, dies umzusetzen (siehe z. B. Österreichische Demenzstrategie, www.demen zstrategie.at).

Die Vulnerabilität von Menschen mit Demenz zeigt sich auf verschiedenen Ebenen. Allein schon die Diagnose ist eine emotionale Herausforderung. Es kann Menschen mit Demenz sehr belasten, wenn sie im frühen Stadium der Demenz selbst merken, dass ihre kognitiven Leistungen stark abnehmen. Viele versuchen dies vor ihren Angehörigen zu kaschieren und sind oft geschickt darin, ihre Vergesslichkeit mit Witzen zu überspielen. Wenn es dann schließlich doch zum Arztbesuch kommt, was häufig von den Angehörigen initiiert wird, ist es für viele Betroffene dennoch schwierig, die Diagnose zu hören. Selbst wenn sie schon damit gerechnet haben, wird die ärztliche Diagnose, tatsächlich an einer Form der Demenz erkrankt zu sein, für viele als Schock empfunden. Auch die Angehörigen sind häufig überfordert, wissen möglicherweise nicht viel über Demenzen und müssen sich auch erst damit zurechtfinden und auseinandersetzen (Schmidhuber/Gräßel 2018).

Vulnerabel sind Menschen mit Demenz auch deshalb, weil sie nach der Diagnose mit einer Stigmatisierung rechnen müssen. Sie werden möglicherweise von ihrem Umfeld nicht mehr ernst genommen, paternalisiert und infantilisiert (Schweda/Jongsma 2018). Dass sie jedoch noch lange ihre Wünsche und Bedürfnisse trotz Demenz äußern können und wollen, wird dabei übersehen. Damit sinkt ihre Lebensqualität, sie fühlen sich unverstanden, können dies aber möglicherweise nicht mehr gut verbalisieren und werden deshalb aggressiv - man spricht in diesem Zusammenhang von „herausforderndem Verhalten“. Diese Aggression spüren wiederum die Angehörigen, was sie verständlicherweise überfordern kann. Selbsthilfegruppen für pflegende Angehörige und auch Freizeitaktivitätsgruppen, in denen sie die Möglichkeit haben, abzuschalten und für sich selbst etwas Gutes zu tun, sind deshalb ein wichtiger Bestandteil für ihre Lebensqualität, da sich ihr Alltag vor allem um die betreuungsbedürftige Person dreht (Voß 2015).

Bei einer Demenz kommen im Krankheitsverlauf häufig auch psychiatrische Begleitsymptome dazu. So fühlen sich die betroffenen Menschen ausgerechnet von jenen Personen, die sie betreuen und pflegen, betrogen oder bestohlen. Das ist besonders schmerzhaft für die Angehörigen, aber auch für professionell Pflegende in einem Heim nicht einfach. Wenn nun aufgrund der Corona-Krise Menschen mit Demenz in ihren Aktivitäten im Heim stärker eingeschränkt werden, kann dies aufgrund der psychiatrisch- 
en Symptome sehr problematisch werden. Ungerechte Behandlung im Heim oder die Unterstellung, die eigenen Kinder wollten oder dürften sie nicht mehr besuchen, können hier zum Vorwurf werden und große Unruhe hervorrufen. Selbst wenn unter diesen Umständen möglichst gute Lösungen gefunden wurden, wie etwa ein Besuch mit Abstand über den Gartenzaun oder vom Balkon, fehlten Berührungen und Umarmungen.

Pflegende Angehörige, die in diesen Krisenzeiten zu Hause einen Menschen mit Demenz betreuen, sind besonders belastet. Wie oben erläutert, ist es für sie besonders wichtig, auch außerhalb des Hauses anderen Tätigkeiten mit kognitiv gesunden Menschen nachgehen zu können. Fällt dies weg, sind der Druck und die Belastung enorm. Die Möglichkeit, sich über digitale Medien auszutauschen, ist eine Zwischenlösung, aber kein adäquater Ersatz, weil dabei viel an Zwischenmenschlichem verloren geht.

\section{Zur Vereinbarkeit der Rückkehr in den Alltag mit dem Schutz vulnerabler Gruppen}

Häufig wurde gerade am Anfang der Krise von politischer Seite mit der Solidarität der Jüngeren gegenüber den Älteren bzw. den Vulnerablen argumentiert. Rückblickend wird Kritik laut, dass der Schutz einer kleineren vulnerablen Gruppe die Existenz einer viel größeren Gruppe in der Gesellschaft gefährdet hat. Menschen wurden arbeitslos und kleinere Unternehmen, die schon vor der Krise vor der Herausforderung standen, irgendwie zu überleben, müssen nun mit Konkurs rechnen, was wiederum auch Arbeitsplätze kostet. Die Existenzgefährdung lässt Menschen, denen es bisher wirtschaftlich gut ging, situativ vulnerabel werden. Aus dieser wirtschaftlichen Perspektive schien es Anfang Mai 2020 höchste Zeit zu sein, wieder in eine Form der Normalität zurückzukehren, die nicht noch mehr ökonomischen Schaden anrichtet.

Kritische Stimmen gaben allerdings zu bedenken, dass ein Wiederansteigen der Ansteckungskurve dadurch nicht auszuschließen sei und dadurch wiederum die vulnerablen Personen besonders gefährdet werden. Im alltagsmoralischen Verständnis wird Gesundheit häufig als das höchste Gut wahrgenommen. Aus dieser Sicht scheint es auf den ersten Blick unangemessen, dieses hohe - wenn nicht sogar höchste - Gut gegen wirtschaftliche Interessen abzuwägen. Gesundheit ist jedoch, folgt man Martha Nussbaums Konzeption des guten Lebens (Nussbaum 1999), nur ein Aspekt des guten Lebens neben vielen anderen, die der Staat zu fördern hat, wie etwa die Verbundenheit mit anderen Menschen. 
In diesem Zusammenhang darf auch nicht übersehen werden, dass Leid nicht nur durch Krankheit hervorgerufen wird, sondern auch durch Arbeitslosigkeit und Armut, und auch dies wiederum Studien zufolge krank machen kann - sowohl physisch als auch psychisch (Schenk/Moser 2010). Problematisch dabei ist, dass ohnehin jene in der Krise benachteiligt sind, die weniger gut ausgebildet sind, es sei denn, sie sind in einem systemerhaltenden Beruf tätig wie etwa im Lebensmittelhandel. Wenn aber jemand beispielsweise in einem Möbelhaus als VerkäuferIn tätig war, dabei ohnehin nicht viel verdient hat, möglicherweise monatlich aufgrund der Wohnungsmiete und der Versorgung der Familie nur knapp mit dem Einkommen auskommt, ist der Verlust des Arbeitsplatzes eine Katastrophe. Zudem ist zu bedenken, dass laut einer Statistik der Österreichischen Nationalbank von 2018 ein Drittel der privaten Haushalte in Österreich verschuldet ist. Die betroffenen Personen rechnen mit ihrem regelmäßigen, gleichbleibenden Einkommen, um etwa den Kredit für eine Eigentumswohnung zurückzahlen zu können. All diese Einzelschicksale sind zu berücksichtigen, bevor man allzu leichtfertig der Wirtschaft den Rang nach der Gesundheit zuteilt.

Im öffentlichen Diskurs wird Kritik laut, wenn von politischer Seite angekündigt wird, Unternehmen finanziell unterstützen $\mathrm{zu}$ wollen. Wo bleibt die ebenso großzügige Unterstützung für die ArbeitnehmerInnen? In diesem Zusammenhang darf nicht vergessen werden, dass es gerade die Kleinunternehmen sind, die Arbeitsplätze schaffen, die aber häufig auch von Monat zu Monat wirtschaften (müssen). In diversen Medienberichten wurden Schicksale von kleinen Unternehmen gezeigt, die eben nicht einfach ein paar Wochen entspannt zusperren können. Auch das Wiederaufsperren kleinerer Geschäfte nach Ostern war für manche Kleinunternehmen nicht ertragreich: Die Miete für die Geschäftsräume war zu bezahlen, die Ware wurde geliefert, aber die Kunden blieben zum Teil aus, weil offenbar wenig Bedarf vorhanden war, etwa Kleider und Schuhe in Krisenzeiten zu kaufen. Auch hier stehen menschliche Schicksale dahinter, die mit viel Arbeit, Mühe und Herzblut ein kleines Geschäft oder ein Lokal führen, dessen Zukunft - und damit auch die persönliche Zukunft dieser Menschen - ungewiss ist.

Gesundheit und Wirtschaft dürfen nicht gegeneinander ausgespielt werden. Beides ist wichtig, gehört zu einem guten Leben und hängt, wie eben gezeigt wurde, auch zusammen. Deshalb war es der richtige Weg, die österreichische Wirtschaft wieder zum Leben zu erwecken. Trotzdem bleibt zu fragen, wie es gleichzeitig möglich ist, die Ansteckungen weiterhin möglichst gering zu halten. 
In Schweden wurden bereits am Anfang der Pandemie ältere Menschen und Menschen mit chronischen Erkrankungen ersucht, zu Hause zu bleiben, damit das „normale“ Leben für die meisten schwedischen BürgerInnen weitergehen konnte. Wenn dies auch so in Österreich gehandhabt wird, wäre es einerseits möglich, dass die meisten Menschen wieder ihrem Beruf nachgehen können, normaler Schulbetrieb wieder umsetzbar ist und die Wirtschaft angekurbelt wird - wenn auch höchstwahrscheinlich sehr langsam, wie ExpertInnen prognostizieren. Andererseits wären die vulnerablen Personengruppen trotzdem geschützt, wenn sie sich dessen bewusst sind, dass sie gefährdet sind und im Sinne ihrer Selbstverantwortung agieren und Kontakte meiden. Das muss freilich auch der/die ArbeitgeberIn unterstützen und z.B. weiterhin Home-Office zugestehen, wenn es etwa um jüngere Menschen mit Diabetes geht, die mitten im Beruf stehen.

$\mathrm{Zu}$ bedenken ist allerdings, dass insbesondere ältere Menschen an Einsamkeit leiden können, vor allem wenn sie alleine wohnen. Auch Einsamkeit macht krank, wie der Psychiater Manfred Spitzer konstatiert (2018). Hier kann tatsächlich Digitalisierung fürs Erste (!) weiterhelfen: das Skypen mit den Enkelkindern, das Schreiben von SMS mit Freunden und Verwandten - jene, die dies aufgrund der weiten Entfernung ihrer Familien nicht ohnehin schon so betrieben haben, können diese Variante in Zeiten wie diesen nutzen. Dass Besuche in Seniorenheimen wieder möglich sind, wenn auch mit einer Glasscheibe als Schutz zwischen den Personen, ist auch ein Schritt in die richtige Richtung.

\section{Zur Notwendigkeit spezieller Angebote für vulnerable Gruppen in der Krise}

Menschen mit Demenz, die diese herausfordernde Situation aufgrund ihrer abnehmenden kognitiven Fähigkeiten gar nicht mehr verstehen können, haben es hier freilich besonders schwer. Das Gefühl, dass etwas nicht stimmt, gleichzeitig das Nicht-verstehen-Können, kann Menschen mit Demenz noch verwirrter und auch depressiv werden lassen und ebenso herausforderndes Verhalten in Form von Aggressionen hervorrufen. Eine noch wichtigere Rolle als im „Normalzustand“ spielen nun die Pflegenden. Die Tatsache, dass pflegende Angehörige und professionell Pflegende nun noch stärker gefordert sind, als dies in ihrem Alltag bisher ohnehin schon der Fall war, führt unserer Gesellschaft ungerechte Strukturen deutlicher denn je vor Augen. Jene, die ohnehin schon unter schwierigen Bedingungen ihren Alltag meistern müssen, trifft die Krise mit voller Wucht. Sie sind es, die sich jetzt besonders um Menschen mit Demenz kümmern und selbst mit ihren Bedürfnissen auf der Strecke bleiben. Deshalb kann 
diese Krise auch als Chance begriffen werden, in der Strukturen verändert werden können, die schon seit längerem als nicht zufriedenstellend und ungerecht diagnostiziert wurden. Pflegende Angehörige müssen stärker von staatlicher Seite unterstützt werden, sowohl finanziell als auch durch personelle Unterstützung, wie etwa mobile Pflege. Wie bedeutsam professionelles Pflegepersonal in unserer Gesellschaft ist, dürfte nun allen BürgerInnen bewusst geworden sein. Hier gilt es, den Beruf attraktiver für junge Menschen zu gestalten, indem etwa die schon vor der Krise debattierte 35-Stunden-Woche bei gleichbleibender Bezahlung eingeführt wird und auf diese Weise langfristig dem Pflegenotstand entgegengewirkt wird.

Gesamtgesellschaftlich betrachtet lässt sich konstatieren, dass insgesamt ein Umdenken erforderlich ist: Von einer stark ökonomisierten und rationalisierten Gesellschaft ausgehend, die viele Menschen aufgrund der Schnelllebigkeit außer Atem kommen lässt - was sich in psychischen Erkrankungen wie Burnout spiegelt (Esterbauer et al. 2019) -, können wir diese Krise als Chance begreifen, mit dem Bewusstsein unserer Vulnerabilität etwas entschleunigter unser Leben zu führen. Eine Gesellschaft, in der alles etwas langsamer ablaufen darf, in der sich aber Menschen, die besonders vulnerabel sind, wieder wohler fühlen, klingt aktuell noch wie eine Utopie. Zum jetzigen Zeitpunkt gilt es deshalb, mit den bestehenden Gegebenheiten einen möglichst guten Umgang zu finden, damit sich etwa Menschen mit Demenz wohler fühlen. Aktivierung und Ablenkung sind dabei besonders wesentlich. Ein Vorzeigebeispiel für Aktivierung, das gleichzeitig Ablenkung ermöglicht und Traurigkeit verhindert, ist das MAKS-Konzept, das zur Steigerung der Lebensqualität von Menschen mit Demenz dient. Das Akronym MAKS steht für motorisch, alltagspraktisch, kognitiv und spirituell. Das MAKS-Konzept ist ein Training, das zwei Mal in der Woche durchgeführt werden soll, und beinhaltet z. B. Ballspiele (motorisch), das Einbeziehen der Menschen mit Demenz in die Speisenzubereitung - z. B. Kartoffeln schälen oder Teig rühren - (alltagspraktisch) und leichte Rätsel mit Zahlen und Buchstaben (kognitiv). Ergänzt sollte das Konzept mit Spiritualität werden, das können der sonntägliche Kirchgang sein und das Singen von Kirchenliedern, welche die Menschen meistens sehr lange im Gedächtnis behalten. All diese Tätigkeiten sind auch mit dem geforderten Abstand und Mund-Nasen-Schutz möglich und können viel bewirken, wie die Ergebnisse einer Studie zeigen. So konnte nachgewiesen werden, dass bei diesem Training die üblicherweise nachlassenden Fähigkeiten bei einer Demenz für eine gewisse Zeit erhalten bleiben können, nämlich bis zu zwölf Monate (Gräßel et al. 2011).

Das Einbauen diverser Aktivitäten in den Alltag ist für Menschen mit Demenz nun besonders wichtig, sowohl im Heim als auch zu Hause. Zwar 
kann die Vulnerabilität damit nicht zum Verschwinden gebracht werden, dennoch ist die Ablenkung - für beide Gruppen - wohltuend und hilfreich, um Zeiten wie diese gut durchzustehen. Für Menschen mit Demenz kann die Aktivierung eine Steigerung der Lebensqualität bedeuten. Psychologische Unterstützung für pflegende Angehörige per Telefon oder Skype ist unabdingbar, damit sie sich nicht alleingelassen fühlen. Dann haben sie sogar die Chance, in dieser Krise, bei allen Belastungen, seelisch und geistig zu wachsen (Kruse 2017). Parallel sollten wir als Gesellschaft nicht hoffen, dass es nach der Krise wieder "normal“ weitergeht, sondern sie als Wendepunkt und Chance zum Umdenken und zur Entschleunigung ergreifen, um ein besseres Leben für alle und insbesondere für vulnerable Personengruppen zu ermöglichen.

\section{Literatur}

Esterbauer, Reinhold/Paletta, Andrea/Meer, Julia (Hg.): Der Leib und seine Zeit. Temporale Prozesse des Körpers und deren Dysregulationen im Burnout und bei anderen Leiberfahrungen, Freiburg: Alber 2019.

Gräßel, Elmar et al.: Non-pharmacological, multicomponent group therapy in patients with degenerative dementia: a 12-months randomized controlled trial, in: BMC Medicine 9 (2011) 129; doi: 10.1186/1741-7015-9-129.

Gräßel, Elmar/Niefanger, Dirk: Angehörige erzählen. Vom Umgang mit Demenz: Einige sozialmedizinische und narratologische Beobachtungen, in: Freiburg, Rudolf/Kretzschmar, Dirk (Hg.): Alter(n) in Literatur und Kultur der Gegenwart, Würzburg: Könighausen \& Neumann 2012, 99-116.

Gronemeyer, Reimer: Das 4. Lebensalter. Demenz ist keine Krankheit, München: Pattloch 2013.

Juraszovich, Brigitte/Sax, Gabriele/Rappold, Elisabeth et al. (Hg.): Demenzstrategie: Gut Leben mit Demenz. Bundesministerium für Gesundheit und Sozialministerium, Wien 2015.

Kruse, Andreas: Lebensphase hohes Alter. Verletzlichkeit und Reife, Berlin: Springer 2017.

Mackenzie, Catriona/Rogers, Wendy/Dodds, Susan: Introduction: What is vulnerability and why does it matter for moral theory?, in: Dies. (Hg.): Vulnerability. New Essays in Ethics and Feminist Philosophy, New York: Oxford University Press 2014, 1-29.

Nussbaum, Martha C.: Menschliche Fähigkeiten, weibliche Menschen, in: PauerStuder, Herlinde (Hg.): Gerechtigkeit oder Das gute Leben, Frankfurt/Main: Suhrkamp 1999, 176-226.

Schenk, Martin/Moser, Michaela: Es reicht! Für alle! Wege aus der Armut, Wien: Deuticke 2010. 
Schmidhuber, Martina/Gräßel, Elmar: Zur Vulnerabilität von Menschen mit Demenz und ihren Angehörigen, in: Bergemann, Lutz/Frewer, Andreas (Hg.): Autonomie und Vulnerabilität in der Medizin. Menschenrechte - Ethik - Empowerment, Bielefeld: transcript 2018, 147-166.

Schweda, Mark/Jongsma, Karin: Return to childhood? Against the infantilisation of people with late-onset dementia, in: Bioethics 32/7 (2018) 414-420.

Spitzer, Manfred: Einsamkeit. Die unerkannte Krankheit, München: Droemer 2018.

Voß, Henrike: Die häusliche pflegerische Situation von pflegenden Angehörigen von Menschen mit Demenz im Kontext von sportlicher und körperlicher Aktivität, Masterarbeit: Köln 2015. 
